\title{
An Interactive Cerebral Blood Vessel Exploration System
}

\author{
Anna Puig Dani Tost Isabel Navazo
}

Polytechnical University of Catalonia

\begin{abstract}
An interactive cerebral blood vessel exploration system is described. It has been designed on the basis of neurosurgeon's requirements in order to assist them in the diagnosis of vascular pathologies. The system is based on the construction of a symbolic model of the vascular tree with an automatic identification and labelling of vessel bifurcations, aneurysms and stenoses. It provides several types of visualization: individual MRA slices, MIP, shaded rendering, symbolic scheme and surface reconstruction.
\end{abstract}

Keywords: Volume Modelling and Rendering, Medical Applications, Cerebral Blood Vessel.

\section{Introduction}

More than five percent of persons older than 65 are affected by cerebral vascular diseases, which may cause severe cerebral damage and eventually death. Vascular diseases may be classified into two main groups: embolisms, caused by an obstruction of stenotic vessels, and haemorrhages which appear as a consequence of a vessel rupture due to an aneurysm or local growth of its diameter. An early diagnosis and risk evaluation of vascular anomalies can prevent many diseases, either by effecting changes in the diet and life style of the patients or by surgical operations such as vessel prothesis implant.

Current diagnosis methods are based on vessel images obtained by: X-Rays, DSA (Digital Subtraction Angiography), contrasted CT (Computer Tomography) or MRA (Magnetic Resonance Angiography). From these images physicians must mentally reconstruct the $3 \mathrm{D}$ shape of the vcssels in order to detect lesions. This is a difficult task because the brain vascular system has a complex tree-like structure, and the vessels are sparse in comparison to the surrounding volume.

According to the neurosurgeons consulted, a computerassisted system for the exploration and neurodiagnosis should provide the following functionalities:

- Interactive visualization of the MRA slices such as the one provided by the capture device.

- Visualization of the $3 D$ vascular system in order to allow a good perception of the spatial location of the relevant vessels and their topological connectivity. The vessels of interest are those whose diameter is larger than a given value becanse vascular accidents in capillary vessels do not produce significant injuries.

- Automatic detection of the vessel features. The system must provide a visualization that explicitly indicates stenoses, aneurysms and vessel branching in order to assist the diagnosis.

0-8186-8262-0/97\$10.00 Copyright 1997 IEEE.
- Fly-Navigation inside and outside the vascular system. The user may walkthrough the vascular tree in order to ubtain a complete analysis of the vascular system.

- 3D mixed visualizations of the vessels and the brain. This visualization must show heamorrhages and allow the detection of deformations of the vessels produced by lesions in the parenchyma, such as tumurs.

- Blood flow simulation, in order to predict vascular accidents.

In this paper a Computer Assisted NeuroDiagnosis system based on MRA images is presented that fulfills physician's requirements. Previous works are briefly reviewed in section 2. Section 3 describes the functional diagram of the system and the blood vessel representation schemes: voxels model and symbolic model. In section 4, the algorithm for obtaining the symbolic model is presented. The different visualizations of the system are described in section 5. Finally, an evaluation of a prototype of the application is done in section 6 .

\section{Previous work}

Current MRA-based neurovascular applications may be classified into three categories: direct visualization of the voxel model, blood vessels surface extraction and construction of a symbolic model of the vascular tree. As MRA data are typically distributed along a wide range of values, local classification of the voxels according to their property value is not feasible, and a segmentation preprocess is required for most operations on the data [VVB +94$]$.

The direct visualization is generally performed using the Maximum Intensity Projection (MIP) [GS95]. MIP does not require segmentation but it lacks depth perception, and therefore produces ambiguities in the overlapping of vessels, diameters reduction and loss of the smallest vessels. Depth clues can be added to the rendering [HMS95] but it still cannot provide a perception of the whole $3 D$ vascular tree in a single image: in practice, several different MIP views are computed in batch and recorded as a film for clinician's use, which is computationally expensive. Shaded visualizations require a good segmentation $\left[\mathrm{HBP}^{+} 90\right]$ and are generally two slow for routine diagnosis, although they can be speeded-up by using hardware 3D texture mapping [Zui96].

The narrow tubular structure of the vessels poses numerous problems for the blood vessels surface extraction. Marching Cubes (MC) [GKJ90] and Dividing Cubes (DC) [CLKJ90] algorithms may skip over small vessels and generate a huge amount of polygonal faces. According to [EDKS94], the trilinear interpolation done in these methods is not suitable for the modelling of local deformations in elbows, aneurysms and stenoses.

The construction of a symbolic model of the vascular tree provides a good understanding of the siructure. In addition, it may allow the generation of a simpler and smoother 
surface model, therefore suitable for fast interactive visualizations, navigations and flow simulations. However, it encounters several problems: axes and bifurcation automatic detection, diameters computation. In [BGSC85] a symbolic model of individual vessels was early proposed, composed of cylinders joined onto common circular sections and extracted manually. In [ZJP94] a symbolic tree of the vascular structure is automatically extracted. It is composed of numbered branches connected at bifurcation nodes, where the diameters of the vessels and the axes orientations are stored. The model automatically computed in [EDKS94] is a tree of spline curves marking the center lines of the vessels, plus a sequence of cross-section contours perpendicular to the axis. A smooth surface model composed of tensor product rectangular patches can be fitted on this symbolic model.

The system presented herein extends the idea of the construction of a symbolic model to the identification and modelling of vascular accidents.

\section{Design of the Application}

In order to fulfill the requirements above exposed, the application is based on the functional diagram of figure 1 . A brief description of the different modules follows.

BVinput: This module reads the slices, which are generally a set of 100 images of $256 \times 256$ or $512 \times 512$, spaced $1 \mathrm{~mm}$ apart and with an intensity resolution of 12 bits. These images are stored in a specific data structure for their further process.

BVsegmentation: This module filters the input images and constructs a Voxel Model that stores complete information of the sampled area and indicates explicitly the vessel voxels by setting the voxels to at a value that allows them to be univoquely classified as internal or external. Software based on existing methods is used for this purpose.

BVrecons: This module constructs a symbolic model allowing the recognition of the vessel features and a fast surface reconstruction. It is based on the obtaining of a vessel skeletion as explained in section 4 . The procedure requires a minimum separation between vessels of one voxel.

BVvis1, BVvis2 and BVvis3: These three modules provide different visualizations of the vessel models in order to obtain a complete visual analysis of the vascular system. $B V$ vis 3 is under construction. 'They are explained in detail in section 5 .

BVedit: This module allows an interactive cdition of the symbolic model in order to allow the physician to modify or add extra information to the symbolic model according to his skill and experience.

BVsimul: This module will realize the blood flow simulations. It is currently in development.

The symbolic model is represented by an oriented graph in the direction of the blood flow. The graph nodes represent bifurcations of the vascular system, while the graph edges represent the vessel sections between branchings. Each seclivin is compused of a set of path connected elements of the types shown in figure 2: regular vessels with no anomalies, stenoses and aneurysms. Each of these elements is defined by its type, and their shape is represented by a Generalized Cylinder (GC). A GC is a solid having a 3D curve as a medial line such that at every point of the medial line there is a closed and bounded cross section perpendicular to it.

For our purposes, it is sufficient to store a finite set of selected points of the medial line and their related cross sections. The selected points are such that intermediate cross

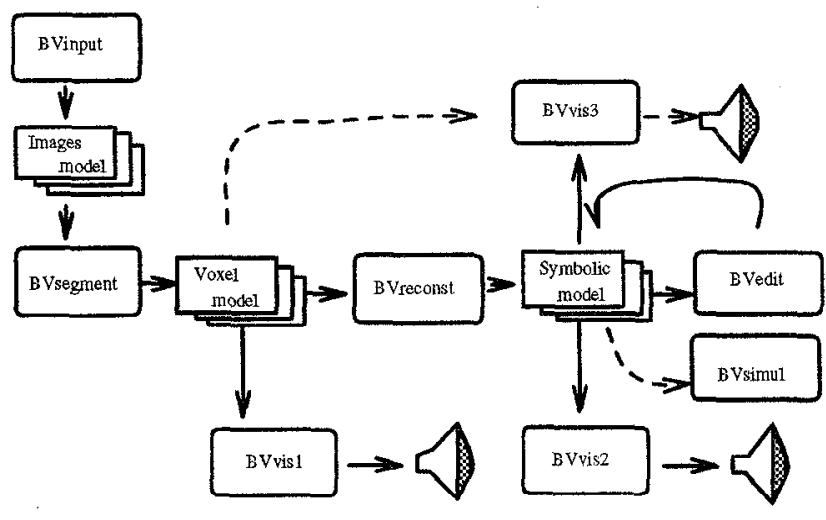

Figure 1: Functional diagram of the application

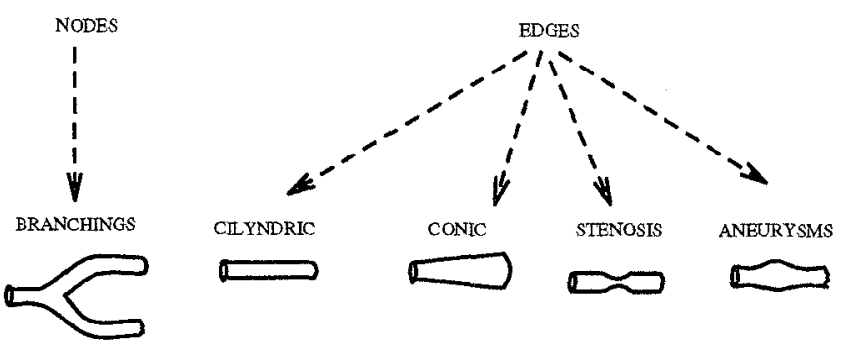

Figure 2: Different types of vascular elements.

sections can be interpolated between them. The shape of the cross sections is approximately elliptic in regular vessels and may be more complex in aneurysms and stenoses. However, it can be considered constant within each element. Therefore, for each element, it is sufficient to store only the medial points and related cross sections where the curvature of the axis changes an amount larger than a specified angle, or where the law defining the cross section area growth changes. This shape representation is valid for regular vessels as well as for anomalies.

The symbolic model synthesizes the characteristics of the physical vessels and represents the topology and the geometry of the vascular system. Moreover, it allows a reconstruction of the vessel surface with different levels of approximation according to the interpolation method used between two consecutive cross sections.

\section{Construction of the symbolic model from the voxel model}

The construction of the blood vessel symbolic model (BVre const) involves two different tasks: first the computation of a discrete skeleton of the blood vessel surface and (2) the identification of the nodes, radii and features from the discrete skeleton along with their topological relationships. Although the two processes are performed simultaneously in a one-pass seed strategy, for clarity they will be explained separately. 


\subsection{Construction of the skeleton}

Skeletal representations liave been extensively studied as simplified shape descriptors [LKC94]. The Medial Axis Transform (MAT) of 2D closed objects is defined [Blu67] as the closure of the set of centers of maximal balls fitted inside the object, not contained in any other ball. DistanceMap skeletons of images derives from the above definition of the MAT, and identifies the centers of discrete maximal discs within discrete figures. Scveral distance-map skeletons algorithms exist, tailored to specific distance definition [Bor84]. Due to the discrete nature of the discs, skeletons defined as the locii of maximal disks centers do not share the homotopy type of the object and therefore exhibit discontinuities [SGP93] which can be reduced by using proper distance functions [Dan80], but not totally avoided.

In order to correctly identify the topological relationships between the vessel tree features and to compute the blood vessel model described in section 3 , a continuous skeleton is required. In [Pui97] a 2D skeletonization distance-map based algorithm is proposed, which provides a path connected discrete skeleton containing all thc centers of the maximal disks along with a minimal set of connecting pixels which guarantee the skeleton continuity. The connecting pixels form minimal arcs and preserve the homotopy of the skeleton with the vessel boundary. The algorithm is based on a growing strategy in which, given a seed pixel of the skeleton trunk, the pixels of its neighbourhood are visited, their distance map are incrementally computed and they are classified either as interior pixels, disks center pixels, or possibly connecting pixels. The algorithm proceeds recursively, taking as seeds the two latter types of pixels. During the process "possibly connecting pixels" are definitively labeled either as belonging to the skeleton or not. The algorithm keeps track of the connected components of the skeleton in order to detect branchings. This algorithm has been extended to 3D [Pui97]. It obtains a skeleton almost linear in regular sections (except for symmetry and parity effects) and in symmetrical aneurysms and stenoses. It forms surfaces in branchings and asymmetrical aneurysms and stenoses.

Colour plates 1 and 2 show the Distance Map and the computed skeleton, respectively, of a 2D case. Colour plate 3 is a zooming image of the previous case in which the skeleton is superposed ontop of the Distance Map.

\subsection{Detection of the features}

The detection of the features, nodes and edges of the symbolic model, is based on the fact that the computed skeleton reflects the shape of the surface.

The skeleton bifurcations are identified by the number of connected component sets of its voxels. These bifurcations may correspond either to real vessel branchings, either to aneurysms or to stenoses. The decision is based on the length of the branch, according to anatomical criteria.

In each branch, the voxels of the skeleton can be classified into two sets: axis-connected and surface-connected voxels. In both cases a vectorization algorithm allows one to obtain the dominant axis. This axis is regularly sampled at a given resolution and a set of cross sections are computed at the sample points. In regular vessels and symmetrical lesions, the cross sections are approximately elliptical and the axis intersection is a segment. The diameters are computed and tracked along the axis to detect changes in the cross section area growth. A sample is only added to the CG axis if a. change in the cross section area growth law is detected or if the axis direction changes. In the same process, if a local minimum or maximum of the ellipse radii is detected, a symmetrical anomaly, stenosis or aneurysm respectively, is labeled in the graph.

In asymmetrical lesions the axis intersection exhibits branchings. The radii associated to the skeleton voxels are computed. The same criterion as for regular vessels is applied to determine which sections should be storcd in the symbolic model. The feature classification is only possible after all the set of cross sections sharing the same topology have been analyzed. It is labeled as stenosis or aneurysm depending on the sign of the radii growth.

This procedure performs a correct labelling in large and medium vessels. However, short vessel branches may be confused with aneurysms and in vessels of small diameter, stenoses and aneurysms, since they are dual, may be misclassified.

\section{Visualization}

As mentioned in section 3 , different visualization strategies are implemented: both of the voxel model (BVVis1) and of the symbolic model (BVVis2). There are three possible visualizations of the voxel model: slice-to slice composition, MIP projection and shaded semi-transparent rendering. The slice-to-slice composition, inspired on the BOB system [CP92], simply overlaps slices of the voxel model parallel to the coordinate plane of maximum projected area, and maps the source pixels onto target pixels with a monochrome level proportional to the property value (see colour plate 4). It allows the composition of the images with transparencies and to project them at different resolutions (blocks of voxels in a slice). A coherent strategy for the replication of source pixels is implemented [LTPN96], which improves the performance of the visualization. Although its quality is insufficient to meet the diagnosis reqnirements, this projection is fast enough to be the default visualization of the system on which the user interacts in order to select the input parameters of the two other voxel visualizations: clipping planes defining the region of interest of the voxel model, active ranges of the property values (i.e. values taken into account in the visualization)) and camera definition. In our experience, clinicians feel comfortable interacting on slices as in conventional $2 \mathrm{D}$ packages.

The MIP projection (see colour plate 5) and the shaded rendering are executed as separate processes within the application in order to reduce waiting time. The shaded rendering is a BTF projection with a coherent splatting strategy improving its performance (EGWM projection [TPN95]). The shading of the internal and external voxels is based on an emission-only model [Max95] while the surface voxels are shaded using a surface scattering model. As a result, the visualization shows a hybrid (surface/volume) aspect.

The symbolic model can be visualized directly, as shown in colour plate 6 , with spheres at the graph nodes and polylines at the branches. The surface model (colour plate 6) is reconstructed as a set of connecting cones between cross sections using spheres in the branchings and aneurysms as in a piping system. It is visualized using polygonal approximations and hardware $Z_{-} B u f f e r$. It is therefore fast and allows navigation inside the pipes.

\section{Conclusions}

A computer assisted cerebral vascular neurodiagnosis system is presented. It is based on the construction of a symbolic 
model of the vascular tree. The construction is not interactive, but its visualization is fast.

The system has been designed following clinician's requirements. At the current stage of its development, a prototype version has becn implemented and successfully expe. rienced by neurologists. The reconstruction of the vascular tree and the automatic detection of anomalies are the most appreciated features of the system. The possibility of manually editing the symbolic model makes clinicians feel more secure. Within the visualization facilities, the navigation is shown to be the more attractive one.

Nevertheless the system has not yet been introduced in a routine diagnosis procedure and therefore is only partly evaluated. This will be the next step of our development.

Future research lines include the design of a mixed visualization of the voxel model simultaneously with the reconstructcd surfacc model (BVvis3), the enhancement of the vessel surface by the use of texture mapping and the use of MRI data along with MRA slices. In addition, the blood flow simulation module (BVsimul) is currently being investigated in collaboration with the Fluid Mechanics Engineering Department of our University.

\section{Acknowledgements}

This work has been done in collaboration with the neurosurgery department of the Hospital Clinico de Barcelona, and with the help of Dr. Royo from the Corachan Clinique. Thanks are due Jordi Garcia, Joaquim Anguas and Jordi Vilanova from our department for their valuable help in the implementation. This work has been partly funded by the Spanish Government grant TIC95-0630-c05-02.

\section{References}

[BGSC85] C. Barillot, B. Gibaud, J. Scarabin, and J. Coatrieux. 3d reconstruction of cerebral blood vessels. IEEE Computer Graphics and Applications, 5(12):13-19, December 1985.

[Blu67] H. Blum. A transformation for extracting new descriptors of shape. Models for the Perception of Speech and Visual Form. MIT Press, Cambridge, Mass., pages 362-380, 1967.

[Bor84] G. Borgefors. Distance transformations in arbritary dimensions. Computer Vision, Graphics and Image Processing, 27:321-345, 1984.

[CLKJ90] H.C. Cline, W.E. Lorensen, R. Kikinis, and F. Jolesz. Three-dimensional segmentation of $\mathrm{mr}$ images of the head using probability and connectivity. Journal of Computer Assisted Tomography, 14(6):1037-104.5, November/Derember 1990.

[CP92] K. Chin-Purcell. Bob: Brick of bytes. AHPCRC, Minnesota SuperComputer Center (Software for Silicon, Graphics), 1992.

[Dan80] P.E. Danielsson. Euclidean distance mapping. Computer Graphics and Image Processing, $14: 227-248,1980$.

[EDKS94] H.H. Ehricke, K. Donner, W. Koller, and W. Straber. Visualization of vasculature from volume data. Compaler and Gruphics, 18(3):395-406, 1994.
[GKJ90] G. Gerig, R. Kikinis, and F.A. Jolesz. Image processing of routine spin-echo mr images to enhance vascular structures: Comparison with $\mathrm{mr}$ angiography. $3 D$ Imaging in Medicine: Algorithms, Systems and Applications, pages 121132, 1990 .

[GS95] Alexandros Savopoulos Georgios Sakas, Marcus Grimm. Optimized maximum intensity projection (mip). 6th Eurographics Workshop on Rendering, pages 81-93, June 1995.

$\left[\mathrm{HBP}^{+}\right.$y0] K.H. Hohne, M. Bomans, A. Pommert, M. Riemer, C. Schierds, U. Tiede, and G. Wiebecke. 3d visualization of tomographic volume data using the generalized voxel-model. The Visual Computer, 6(1):28-36, February 1990.

[HMS95] W. Heidrich, M. McCool, and J. Stevens. Interactive maximum projection volume rendering. Proceedings of the IEEE Volume Visualization 95, pages 11-18, October 1995.

[LKC94] T.C. Lee, R.L. Kashyap, and C.N. Chu. Building skeleton models via $3-d$ medial surface/axis thinning algorithms. CVGIP: Graphical Models and Image Processing, 56(6):462-478, November 1994.

[LTPN96] J. López, D. Tost, A. Puig, and I. Navazo. Voldmi: An open system for volume modeling and visualization. Computer and Graphics, 20(5):703-712, 1996.

[Max95] N. Max. Optical models for direct volume rendering. IEEE Transactions on Visualization and Contuler Graphic's, 1(2):99-108, June 1995.

[Pui97] A. Puig. Contribution to volume modeling and visualization in medicine: Cerebral blood vessels analysis. PhD Extended Abstract, LSI, Polytechnical University of Catalunya, Spain, 1997.

[SGP93] A. Sudhalkar, L. Gursoz, and F. Prinz. Continuous skeletons of discrete objects. ACM Proceedings on Solid Modelling 93, Montreal, Canada, pages 85-94, 1993.

[TPN95] D. Tost, A. Puig, and I. Navazo. A volume visualization algorithm using a coherent extended weigth matrix. Computer and Graphics, $19(1): 37-45,1995$

[VVB $\left.{ }^{+94}\right]$ D. Vandermeulen, R. Verbeeck, L. Berben, D. Delaere, P. Suetens, and G. Marchal. Continuous voxel classification by stochastic relaxation: Theory and application to $\mathrm{mr}$ imaging and $\mathrm{mr}$ angiography. Internal Research Report, Medical Imaging Research Lab, ESAT, Belgium, 1994.

[ZJP94] C. Zahlten, H. Jurgens, and H.O. Peitgen. Reconstruction of branching blood vessels from ctdata. Proceedins of Rostock, 1994.

[Zui96] K.J. Zuiderveld. Vr in radiology - first experiences at the university hospital utrecht. $A C M$ Computer Graphics, pages 47-48, November 1996. 